\title{
Erratum: Extremal and nonextremal Kerr/CFT correspondences
}

\section{S. Carlip}

Department of Physics, University of California

Davis, CA 95616, U.S.A.

E-mail: carlip@physics.ucdavis.edu

ERRATUM TO: JHEP04(2011)076

KeYwords: Black Holes, Models of Quantum Gravity

The full central term in the ADM form of the $(3+1)$-dimensional Einstein-Hilbert action, described in appendix $\mathrm{A}$ of [1], is

$$
\begin{aligned}
K[\xi, \eta]=B\left[\{\xi, \eta\}_{S D}\right] & -\frac{1}{8 \pi G} \int_{\partial \Sigma} d^{2} x \sqrt{\sigma} n^{k}\left[\frac{1}{\sqrt{q}} \pi_{i k}\{\xi, \eta\}_{S D}^{i}-\frac{1}{2} \frac{1}{\sqrt{q}}\left(\hat{\xi}_{k} \eta^{\perp}-\hat{\eta}_{k} \xi^{\perp}\right) \mathcal{H}\right. \\
& +\left(D_{i} \hat{\xi}_{k} D^{i} \eta^{\perp}-D_{i} \hat{\eta}_{k} D^{i} \xi^{\perp}\right)-\left(D_{i} \hat{\xi}^{i} D_{k} \eta^{\perp}-D_{i} \hat{\eta}^{i} D_{k} \xi^{\perp}\right) \\
& \left.+\frac{1}{\sqrt{q}}\left(\hat{\eta}_{k} \pi^{m n} D_{m} \hat{\xi}_{n}-\hat{\xi}_{k} \pi^{m n} D_{m} \hat{\eta}_{n}\right)+\left(\xi^{\perp} \eta^{i}-\eta^{\perp} \xi^{i}\right)^{(3)} R_{i k}\right] .
\end{aligned}
$$

In the published version of the paper, the last term, proportional to ${ }^{(3)} R_{i k}$, was omitted. The omission does not affect the results reported in the paper, for which this term is irrelevant. The missing term is, however, important for certain other configurations, such as the BTZ black hole, as described in [2]

\section{References}

[1] S. Carlip, Extremal and nonextremal Kerr/CFT correspondences, JHEP 04 (2011) 076 [arXiv:1101.5136] [INSPIRE].

[2] S. Carlip, Effective Conformal Descriptions of Black Hole Entropy, arXiv:1107.2678 [INSPIRE]. 